\title{
Improved operative efficiency using a real-time MRI-guided stereotactic platform for laser amygdalohippocampotomy
}

\author{
Allen L. Ho, MD, ${ }^{1}$ Eric S. Sussman, MD, ${ }^{1}$ Arjun V. Pendharkar, MD, ${ }^{1}$ Scheherazade Le, MD, ${ }^{2}$ \\ Alessandra Mantovani, MD, ${ }^{1}$ Alaine C. Keebaugh, PhD, ${ }^{3}$ David R. Drover, MD, ${ }^{4}$ \\ Gerald A. Grant, MD, , Max Wintermark, MD, MAS, MBA, ${ }^{1,5}$ and Casey H. Halpern, MD1,2 \\ Departments of ${ }^{1}$ Neurosurgery, ${ }^{2}$ Neurology, ${ }^{4}$ Anesthesia, and ${ }^{5}$ Radiology, Stanford University, Stanford; and ${ }^{3}$ MRI Interventions, \\ Inc., Irvine, California
}

\begin{abstract}
OBJECTIVE MR-guided laser interstitial thermal therapy (MRgLITT) is a minimally invasive method for thermal destruction of benign or malignant tissue that has been used for selective amygdalohippocampal ablation for the treatment of temporal lobe epilepsy. The authors report their initial experience adopting a real-time MRI-guided stereotactic platform that allows for completion of the entire procedure in the MRI suite.
\end{abstract}

METHODS Between October 2014 and May 2016, 17 patients with mesial temporal sclerosis were selected by a multidisciplinary epilepsy board to undergo a selective amygdalohippocampal ablation for temporal lobe epilepsy using MRgLITT. The first 9 patients underwent standard laser ablation in 2 phases (operating room [OR] and MRI suite), whereas the next 8 patients underwent laser ablation entirely in the MRI suite with the ClearPoint platform. A checklist specific to the real-time MRI-guided laser amydalohippocampal ablation was developed and used for each case. For both cohorts, clinical and operative information, including average case times and accuracy data, was collected and analyzed.

RESULTS There was a learning curve associated with using this real-time MRI-guided system. However, operative times decreased in a linear fashion, as did total anesthesia time. In fact, the total mean patient procedure time was less in the MRI cohort ( $362.8 \pm 86.6$ minutes) than in the OR cohort ( $456.9 \pm 80.7$ minutes). The mean anesthesia time was significantly shorter in the MRI cohort (327.2 \pm 79.9 minutes) than in the OR cohort (435.8 \pm 78.4 minutes, $p=0.02$ ).

CONCLUSIONS The real-time MRI platform for MRgLITT can be adopted in an expedient manner. Completion of MRgLITT entirely in the MRI suite may lead to significant advantages in procedural times.

https://thejns.org/doi/abs/10.3171/2017.1.JNS162046

KEY WORDS laser interstitial thermal therapy; LITT; interventional MRI; laser ablation; checklist; ClearPoint; temporal lobe; amygdalohippocampotomy; epilepsy surgery

$\mathrm{M}$ R-GUIDED laser interstitial thermal therapy (MRgLITT) is a minimally invasive method for thermal destruction of benign or malignant tissue that has been used to treat a variety of neurological pathologies, including tumors, epilepsy, and chronic pain. ${ }^{12,13,17}$ The MRgLITT system delivers low-voltage laser energy via an optical fiber to pathological tissue, where it is converted to thermal energy, inducing cellular injury. The extent of thermal damage is guided by real-time MRI and automated feedback control. Various stereotactic tech- niques are used to accurately deliver the laser probe within the intracranial space. Choice of a platform for stereotactic delivery depends upon institution-dependent factors, physician preference, accuracy/precision tolerances, and requisite patient position or target. ${ }^{4}$ MRgLITT possesses several advantages over traditional anterior temporal lobectomy, including immediate radiographic visualization of treatment effect, decreased perioperative and surgical morbidity, and the promise of a "less-invasive" treatment, which may increase utilization of surgical treatments of

ABBREVIATIONS MRgLITT = MR-guided laser interstitial thermal therapy; MTS = mesial temporal sclerosis; OR = operating room.

SUBMITTED August 5, 2016. ACCEPTED January 5, 2017.

INCLUDE WHEN CITING Published online June 30, 2017; DOI: 10.3171/2017.1.JNS162046. 
epilepsy. Although the clinical efficacy of this relatively novel technique has yet to be tested in a randomized controlled clinical trial, several institutions have adopted MRgLITT for the treatment of epilepsy, given the available promising data. ${ }^{5,13,17}$

At the vast majority of institutions utilizing this technology, the fiberoptic applicator is typically placed under stereotactic guidance in the operating room (OR). The patient is subsequently transported to the MRI suite for verification and activation of the laser-diode energy source and MRI monitoring of the thermal ablation. ${ }^{4}$ Completing a single surgical procedure in multiple treatment rooms is suboptimal. Transporting an anesthetized patient from the OR to the MRI suite can be a logistical obstacle, and this process is fraught with opportunities for complication and error. Furthermore, the transfer process is inevitably associated with longer operative and anesthesia times and increased risk to the patient. ${ }^{1}$

ClearPoint (MRI Interventions) is a real-time MRI platform that allows the entire procedure to be completed in the MRI suite. This novel frameless stereotactic platform utilizes a minimally invasive percutaneous SmartFrame (MRI Interventions) that allows for real-time neuronavigation. ${ }^{8,17}$ There is a well-reported precedent for real-time MRI in deep brain stimulation surgery. In patients with Parkinson's disease and in pediatric patients with dystonia, the real-time MRI platform has produced clinical outcomes and safety profiles similar to those achieved with conventional deep brain stimulation techniques. ${ }^{11,15}$ Nevertheless, to date, this novel technology has not been widely adopted by the neurosurgical community for MRgLITT. This is, at least in part, due to familiarity with existing stereotactic technology and logistical concerns related to implementing a new stereotactic system outside the comfort of the traditional OR setting. As the popularity of and indications for MRgLITT continue to grow, it is essential to critically assess its limitations and obstacles and identify modifications that may optimize the efficacy and efficiency of this technology.

Here, we review our experience with 2 stereotactic platforms for laser fiber placement: 1) the Vertek Biopsy Solution (Medtronic), used in the OR; and 2) the real-time MRI system, used in the MRI suite. At our institution, the Vertek Biopsy Solution has been used not only for stereotactic laser placement but also for brain biopsies and depth electrode placement. ${ }^{10}$ Thus, there is great familiarity with this option, which was used in the first MRgLITT cases performed at Stanford University. ${ }^{4}$ However, we subsequently adopted the real-time MRI system based on the hypothesis that a dedicated real-time MRI solution would be more efficient and patient-oriented. Indeed, the actual ablation for LITT must be performed in an MRI unit, thus the procedure already requires the use of a diagnostic scanner. Given the novelty of this approach, we designed a checklist for the real-time MRI system based on our initial experience. Deployment of checklists in medicine has been effective for increasing efficiency and minimizing error rates. ${ }^{3}$ This has been demonstrated specifically within step-heavy neurosurgical procedures with an emphasis on stereotaxy.,2,19 The following study is designed to assess operative efficiency in the adoption of real-time
MRI in stereotactic laser placement for MRgLITT for the treatment of patients with temporal lobe epilepsy.

\section{Methods \\ Patient Selection}

Between October 2014 and May 2016, 17 consecutive patents were selected by a multidisciplinary epilepsy board consisting of neurosurgeons, neurologists, neuropsychologists, and neuroradiologists to undergo a functional selective amygdalohippocampotomy via laser ablation. The procedure was performed using the Visualase Thermal Ablation System (Medtronic) at Stanford Health Care. These cases were conducted at an academic center where resident and fellow training is prioritized and incorporated into routine cases. The neurosurgical team was led by the senior author (C.H.H.), who was the attending neurosurgeon of record for each case. Anesthesia and surgical personnel were rotated on a case-by-case basis, and only the attending surgeon (C.H.H.) was maintained throughout every procedure. This study was performed at Stanford Hospital with approval from the Stanford University Internal Review Board, and Stanford's Infection Control approved the performance of this procedure within the inpatient diagnostic MRI suite.

\section{OR Stereotaxy}

The first 9 patients underwent standard laser ablation in 2 phases. ${ }^{4,12}$ Skull fiducial placement and stereotactic CT acquisition were completed either prior to the day of the operation or before the patients entered the OR. After the patients entered the OR, general anesthesia was induced, their head was fixed in MRI-compatible Mayfield pins (Integra LifeSciences), and they were placed in the prone position. After frameless stereotactic registration using StealthStation and the Vertek Biopsy Solution (Medtronic), a small incision and twist drill bur hole were planned and placed using the precision aiming device, and the LITT laser was positioned in the planned target. ${ }^{9}$ An O-arm (Medtronic) was used to confirm optimal placement before transporting to MRI. Skull fiducials were removed and the patients were subsequently transported to the MRI suite, where a laser amygdalohippocampotomy was performed.

\section{Real-Time MRI Stereotaxy}

The next 8 patients underwent laser ablation entirely in the MRI suite facilitated by the ClearPoint platform. ClearPoint is a novel stereotactic implantation system consisting of an MRI-compatible percutaneous SMARTframe, an MRI-compatible hand-held twist drill, a ceramic stylet, and a trajectory guide tower as well as device-specific control software that allows stereotactic placement of the laser entirely in the MRI suite, thus obviating the need for skull fiducial markers or a stereotactic CT scan. ${ }^{17,18}$ All components needed for the stereotaxy are included in the ClearPoint disposable kit (Item No. CP-NGS-11-TE-TP45). At our institution, the diagnostic MRI suite antechamber is frequently used for general endotracheal anesthesia induction (i.e., for patients who cannot tolerate awake MRI scans). There is back-up anesthesia equipment available, 


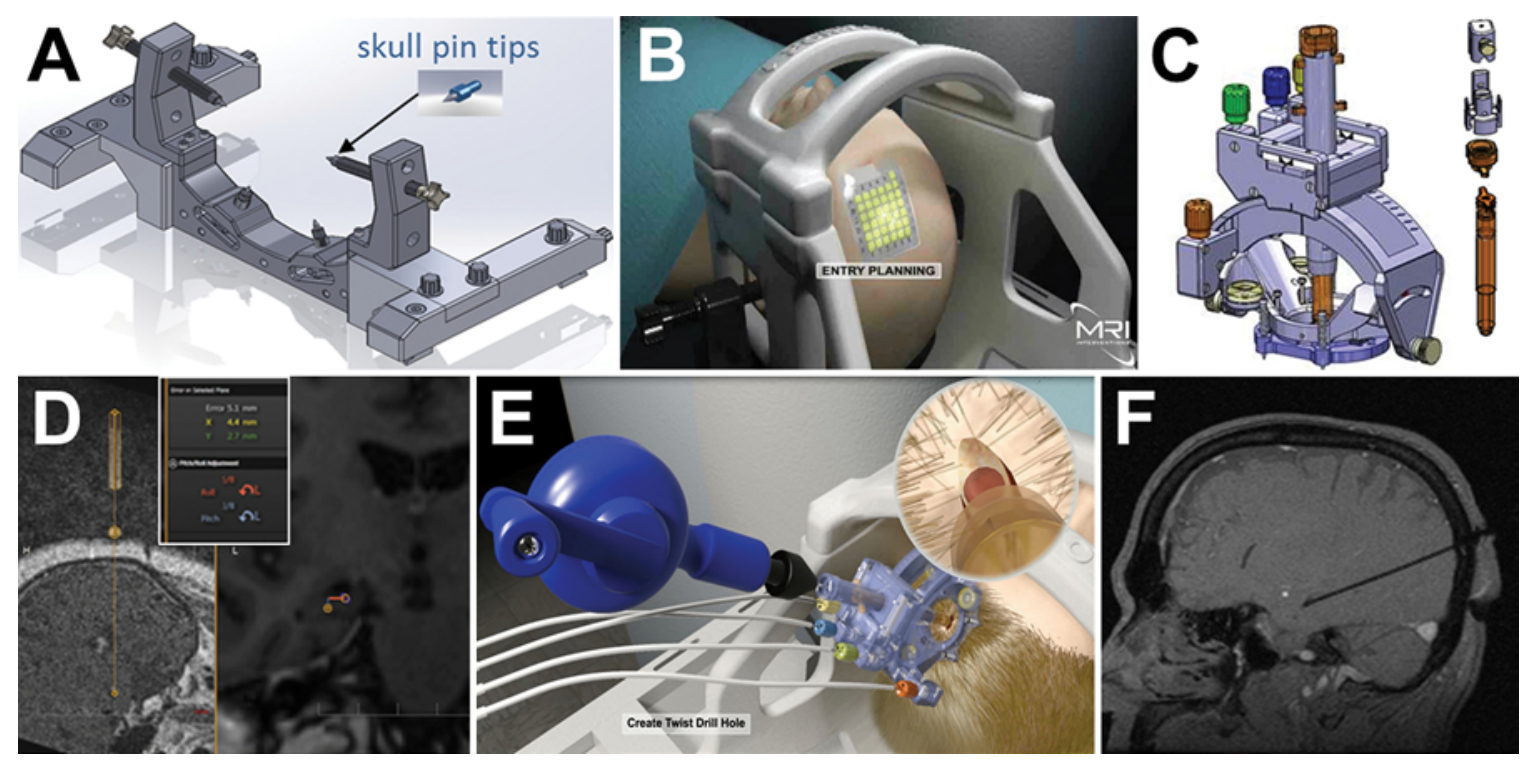

FIG. 1. Real-time MRI workflow and equipment. A: ClearPoint 4-point fixation headframe. B: MRI-visible SmartGrid. The SmartGrid is applied to the predicted skull entry point and secured with adhesive and staples. C: Percutaneous SmartFrame mount. D: T1-weighted volumetric MR images. The MR images are obtained with MR-visible fiducial markers in place in order to allow for precise microadjustments to the SmartFrame. E: MRI-compatible handheld twist drill. The drill is used to create a 3.2-mm bur hole. F: Sagittal T1-weighted MR image obtained after placement of the 2.1-mm ceramic stylet, confirming the desired trajectory. Figure is available in color online only.

including a ventilator, and it is fully staffed by anesthesia personnel (including back-up anesthesiologists) similar to any other OR. Both anesthesia and surgical personnel, with the exception of the senior author (C.H.H.), were rotated and different for each procedure (i.e., there was no dedicated anesthesia or surgical team for these cases). The MRI suite is also commonly used for other sterile surgical procedures at our institution. Thus, we used standard protocols approved by Stanford Infection Control to terminally clean and sterilize the MRI suite before and after each procedure, as in any OR. Patients were brought directly to the antechamber of the MRI suite where general anesthesia was induced. These patients were then positioned prone in the ClearPoint 4-point fixation headframe (Fig. 1A) specifically manufactured for intraoperative MRI and draped in a sterile fashion with a specialized drape that completely covers the internal components of the MRI bore (MR Neuro Drape Tapered with Extension-Long, Product No. NGS-PD-03-L, MRI Interventions). A ClearPoint MRI-visible SmartGrid was applied to the predicted skull entry point, secured with adhesive and staples, and a T1-weighted volumetric image was obtained (Fig. 1B). The ClearPoint software was then used to mark the planned entry point using a gadolinium-filled scalp fiducial marker to guide mounting of the percutaneous SmartFrame around the confirmed entry point (Fig. 1C). A second MRI scan was completed to confirm optimal entry and target error. The ClearPoint software uses MR-visible fiducial markers to allow for precise microadjustments to the SmartFrame (Fig. 1D). An MRI-compatible hand-held twist drill included in the kit was used to create a $3.2-\mathrm{mm}$ bur hole (Fig. 1E). A 2.1-mm ceramic stylet from the kit was then advanced to $50 \mathrm{~mm}$ above the target (Fig. 1F). A final MRI scan confirmed the desired trajectory, and the laser was advanced for the amygdalohippocampotomy. ${ }^{17,18}$

\section{Laser Ablation}

The laser ablation portion of the cases in both the OR and the MRI cohort were completely identical in terms of technique. We used the Medtronic Visualase thermal therapy system Model VTTS-15 with software version 3.2. The principles and aim of laser ablation were adopted from previously described techniques to ensure full ablation of mesial structures from the anterior amygdala to the posterior hippocampus at the level of the tectum. ${ }^{17}$

\section{Patient Demographics and Data Collection}

For both cohorts, patient demographic and clinical data were collected, including age, sex, and target. Complications related to the procedure and seizure-related clinical outcomes were also collected. Furthermore, intraoperative times and all catheter manipulation including repositioning were recorded for every step of each procedure. Finally, for the real-time MRI cohort, the 2D radial errors were defined as the distance between the planned target and the intersection of the device axis with the target plane and were calculated within the ClearPoint software (Fig. 2).

\section{Checklist}

As described previously for deep brain stimulation surgery, ${ }^{6,7}$ a checklist specific to the real-time MRI-guided laser amydalohippocampal ablation was designed by our industry collaborator as well as the first and senior authors (A.L.H. and C.H.H.) and refined in conjunction with the multidisciplinary anesthesia, surgical, nursing, and radiol- 


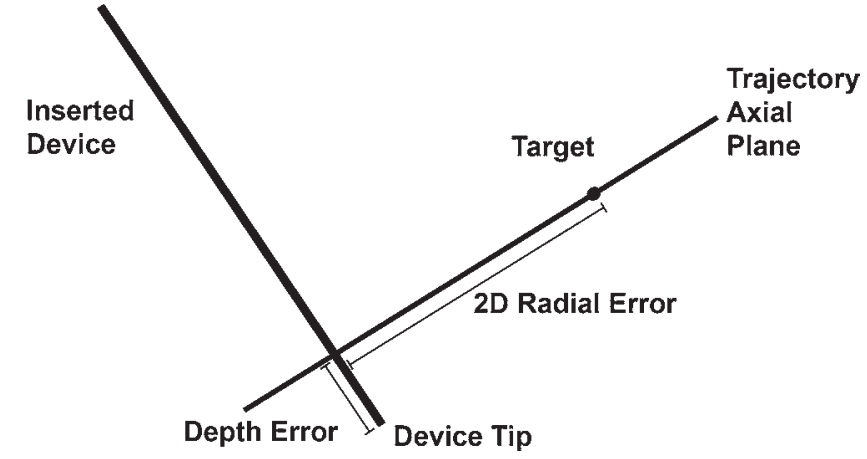

FIG. 2. The $2 \mathrm{D}$ radial error is the distance between the planned target and the intersection of the device axis with the target plane and was measured in millimeters. ogy team members (Fig. 3). Each item on the checklist was verified by the lead surgeon (C.H.H.) and served dual purposes of creating consistency across teams and ensuring step-wise progression through the case without skipping any critical steps. Time points were noted and recorded for each phase of the case by an objective observer. We did not include a checklist for the initial OR cohort as the surgical technique used was identical in both cohorts and common to our practice of stereotactic depth electrode placement and biopsy protocols.

\section{Results}

\section{Patient Demographics and Clinical Outcomes}

The mean age was comparable between the 2 cohorts (46.9 vs 42.1 years for OR and MRI, respectively). The 2

\section{Procedure Checklist}

\begin{tabular}{|l|l|}
\hline In/Out & $\begin{array}{c}\text { Today's } \\
\text { Case }\end{array}$ \\
\hline Pin to Insert & \\
\hline $\begin{array}{l}\text { Pin to Laser } \\
\text { confirmation }\end{array}$ & \\
\hline
\end{tabular}

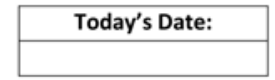

Patient Arrival Time:
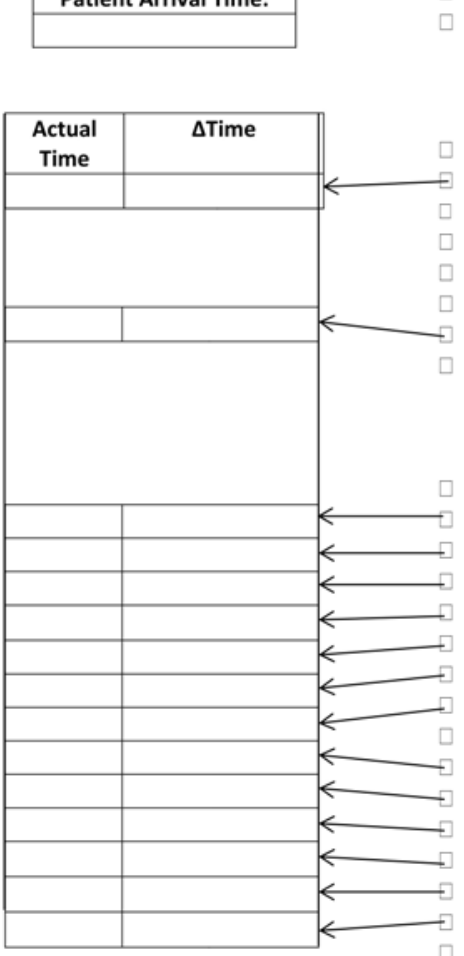

\section{Day before surgery:}

Confirm patient does not have a VNS implant

MRI staff confirm network connection and retrieve preplanning image

\section{Prior to sterile setup/opening case pack:}

Preplan using patient's most recent MRI study w/contrast if available Confirm terminal clean done, room swept and clean

Case cart present and stocked, with instruments present and sterile Confirm present: Mayo stand, garbage can, mobile light, stepstool

ClearPoint and Visualase disposables present

MRI tech present, scanner working, intercom working

Patient MRI metal checklist done and in Epic

MRI staff present, system checked (network connection and mirror screen)

MRI head holder and pins ready, flexible coil covered and ready

\section{Prepping the Patient:}

Anesthesia monitors working, cart stocked, gases and ventilator working Patient transferred to MRI table

Patient asleep and intubated

Foley (metal clip removed)

Compression stockings, socks, SCDs on

Patient ear plugs in

MRI-compatible temperature probe placed

Patient pinned

\section{ClearPoint/Visualase Component:}

ETT and lines tucked, taped and confirmed at isocenter and surgicenter

Localizer and asset calibration scans

Prep, drape, apply marking grid

T1+contrast volumetric scan, begin initial planning

Awl mark, remove grid, Beekley marker confirmation

Mount frame and align by hand to get close

T1 frame scan, confirm trajectory

Alignment scans and adjustments complete

Timeout, Local, incision, and drilling

Measure and mark ceramic stylet

Insert ceramic stylet, scan to confirm placement

Measure and insert catheter with titanium stiffening stylet

MRI verification of laser placement

Begin ablating

Remove frame

Patient out

FIG. 3. Real-time MRI procedure checklist. ETT = endotracheal tube; $S C D$ = sequential compression device; VNS = vagus nerve stimulation. 
TABLE 1. Comparison of mean procedure times in the OR and real-time MRI cohorts

\begin{tabular}{lccl}
\hline \multirow{2}{*}{ Variable } & \multicolumn{2}{c}{ Cohort } & \\
\cline { 2 - 3 } & OR & MRI & p Value \\
\hline No. of cases & 9 & 8 & \\
\hline Target & & & \\
\hline Rt & 4 & 6 & \\
\hline Lt & 5 & 2 & \\
\hline Case times in mins & & & \\
\hline Total & 456.9 & 362.8 & 0.057 \\
\hline Anesthesia & 435.8 & 327.3 & 0.023 \\
\hline Pin to laser & 253.7 & 191.4 & 0.0061 \\
\hline MRI time & 185 & 362.8 & 0.0013 \\
\hline
\end{tabular}

groups included a total of 7 women, 5 in the OR group and 2 in the MRI group. Ten of 17 procedures were right-sided, with 4 right-sided procedures performed in the OR cohort and 6 right-sided procedures in the MRI cohort (Table 1). All patients were treated with a laser amygdalohippocampotomy. Postoperative complications in the OR cohort included a transient partial third cranial nerve palsy and aseptic meningitis with status migrainosus (in the same patient), a self-limited ileus, and persistent headaches in a single patient with a prior migraine disorder. There were no postoperative complications in the MRI cohort. Regarding postoperative seizures, 1 patient within each cohort had a seizure within 3 months of the procedure; all other patients remained seizure-free for up to 3 months.

\section{Case Times}

A comparison of the mean case times for the 2 stereotactic methods is shown in Table 1 . The mean total case time, mean total anesthesia time, and mean pin to laser time were all less when using the real-time MRI system. Total case time refers to time from the moment the patient enters the OR or MRI suite until the patient is taken to the postanesthesia case unit. Total anesthesia time refers to the time from induction of anesthesia to extubation. Pin to laser time spans the period from the beginning of pin placement to confirmation of laser placement via MRI. This also includes the time needed for repositioning when necessary. Total MRI time refers to the total length of time patients spent in the MRI suite for both the OR and the real-time MRI cohort.

There was no clear trend associated with case times in the OR cohort (Fig. 4). With some expected variance, the mean total case time and mean total anesthesia time for the OR cohort were 456.9 minutes and 435.8 minutes, respectively (Table 1). There was a learning curve associated with using the real-time MRI system (Fig. 5). This curve was apparent for total case times, total anesthesia times, and pin to laser time, with strong associations present between total case and total anesthesia times (Pearson coefficient $0.99, \mathrm{p}<0.0001$ ) and pin to laser times (Pearson coefficient $0.93, \mathrm{p}=0.0007$ ). There was no clear trend in laser ablation times in the MRI cohort, and no association between total case and laser ablation times (Pearson

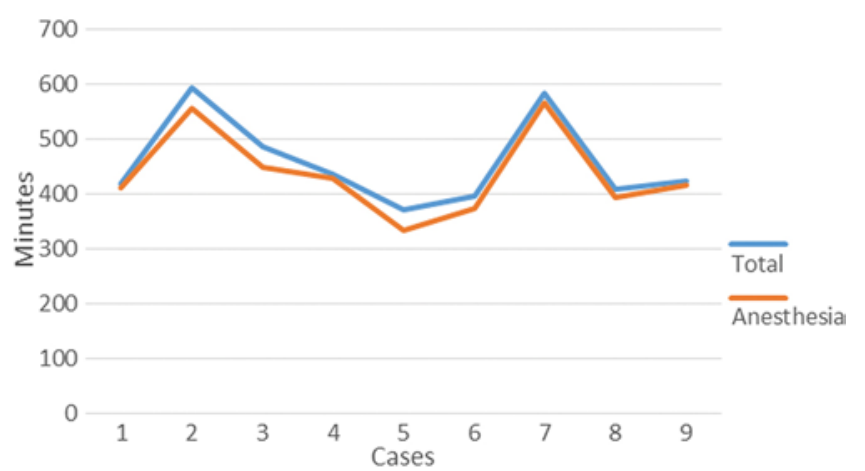

FIG. 4. Standard OR cohort case times. Longitudinal line-graph representation of changes in case times (in minutes) over time for cases with laser placement completed in the OR. Figure is available in color online only.

coefficient 0.097, $\mathrm{p}=0.8189$ ) in these patients (Fig. 5). Case times for each of the patients in the MRI cohort are listed in Table 2. Operative times decreased in a linear fashion nearly $50 \%$ from Case 1 to Case 8 in terms of total patient time as well as anesthesia time. From a procedural standpoint, there was a $44 \%$ decrease in the pin to laser time. Mean operative and mean total anesthesia times for the MRI cohort compared favorably against precursor OR cases. In fact, total case time decreased from a mean of 456.9 minutes in the OR cohort to 362.8 minutes in the MRI cohort, a difference that approached statistical significance $(p=0.057)$. For total anesthesia time, there was a statistically significant decrease in the MRI cohort (mean 327.3 minutes) in comparison with the OR cohort (435.8 minutes, $p=0.023$ ). There was also a statistically significant decrease in pin to laser time in the MRI cohort ( $\mathrm{p}=$ 0.0061 ), despite an expected significant increase in total MRI time in the MRI cohort $(\mathrm{p}=0.013)$ (Table 1$)$.

It should be noted that only 1 real-time MRI case required multiple trajectories, needing 45 additional minutes. If the time for repositioning is not included, Case 1 still had the longest overall case times-495 minutes total case time and 450 minutes total anesthesia time. When analyzing what factors accounted for the difference in time between cohorts, we found that there was an expected and necessary delay in the OR associated with mobilizing the

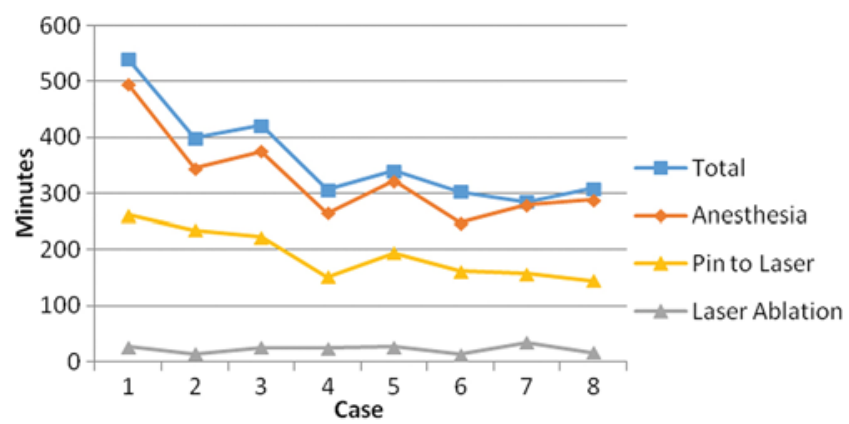

FIG. 5. Real-time MRI case times. Longitudinal line-graph representation of changes in case times (in minutes) over time for cases completed completely within the MRI suite. Figure is available in color online only. 
TABLE 2. Individual case times and accuracy data for all 8 realtime MRI cases

\begin{tabular}{|c|c|c|c|c|c|c|}
\hline \multirow[b]{2}{*}{$\begin{array}{c}\text { Case } \\
\text { No. }\end{array}$} & \multirow[b]{2}{*}{ Target } & \multicolumn{3}{|c|}{ Case Times in Mins } & \multirow{2}{*}{$\begin{array}{l}\text { Target } \\
\text { Error } \\
(\mathrm{mm})^{*}\end{array}$} & \multirow[b]{2}{*}{ Reposition } \\
\hline & & Total & Anesthesia & $\begin{array}{l}\text { Pin to } \\
\text { Laser }\end{array}$ & & \\
\hline 1 & Rt AHC & 540 & 495 & 261 & 0.9 & 1 \\
\hline 2 & Rt AHC & 399 & 345 & 235 & 0.8 & 0 \\
\hline 3 & Lt AHC & 421 & 375 & 223 & 1.0 & 0 \\
\hline 4 & Lt AHC & 306 & 265 & 152 & 0.8 & 0 \\
\hline 5 & Rt AHC & 340 & 322 & 195 & 0.70 & 0 \\
\hline 6 & Rt AHC & 303 & 248 & 162 & 0.7 & 0 \\
\hline 7 & Rt AHC & 285 & 279 & 158 & 3.4 & 0 \\
\hline 8 & Rt AHC & 308 & 289 & 145 & 1.2 & 0 \\
\hline
\end{tabular}

$\mathrm{AHC}=$ amygdalohippocampotomy.

* Target error was defined as the 2D radial error (Fig. 1) and calculated in millimeters.

patient from OR to the MRI suite. Our mean pin to Oarm laser confirmation time was 165.4 minutes (SD 56 minutes) for the OR cohort, which is similar to what we found in our MRI cohort (191.4 minutes, SD 44 minutes, $\mathrm{p}=0.31$ ). There was a mean additional time of 89 minutes (SD 35 minutes) from OR laser confirmation to MRI laser confirmation and subsequent ablation, representing this patient mobilization delay. Finally, it should be mentioned that there was little variance in the time from the beginning of anesthesia to completion of pin placement in our MRI cohort (average 23 minutes, range 12-35 minutes).

\section{Target Error}

Table 2 reports the 2D radial errors for all MRI cases. Case 1 required a repositioning of the trajectory and the 0.9-mm target error reported reflects the error calculated after repositioning. The highest target error occurred in Case 7, with a 3.4-mm error. As described previously in the Methods, this error is calculated by comparing the planned trajectory to the actual position of the laser. Though there was a discrepancy with the actual trajectory from the plan, we were still able to complete a full ablation as described previously, ${ }^{17}$ so a repositioning in Case 7 was unnecessary.

\section{Discussion}

While traditionally the workflow for LITT involved stereotactic placement of the laser in the OR and then either patient transfer to an MRI scanner ${ }^{4}$ or utilization of intraoperative MRI, ${ }^{16}$ we believe there are advantages to performing the entire procedure in the MRI suite. We examined our initial institutional experience with the realtime MRI system for laser amygdalohippocampal ablation and found that the initial learning curve can be quickly mastered. Moreover, completion of the entire procedure in the MRI suite leads to at least comparable if not improved efficiency, as seen with the statistically significant improvement in procedure times, including total anesthesia time, in our MRI cohort. The most striking result from our study is that all procedure times dramatically and quickly improved during the initial experience of MRgLITT uti- lizing the real-time MRI platform. With the implementation of a checklist, we were able to keep this step-heavy procedure moving forward with confidence that no critical portions of the case were being overlooked. ${ }^{6}$ We could also identify procedural bottlenecks and think critically about how to improve efficiency during these steps. For example, as these were the first cases of their kind at our institution, the checklist ensured that OR and MRI staff had all of the necessary additional equipment and disposables in the MRI suite via steps 3-5 and 9 of the "Prior to sterile setup/ opening case pack" section. This avoided substantial delay had it been necessary to bring missing supplies from the OR to the MRI suite. In addition, given the relative novelty of the ClearPoint 4-point fixation headframe setup for our team, the "Prepping the Patient" section (i.e., Foley catheter, sequential compression devices, ear plugs) ensured we followed every preventive step during this process. Moreover, the surgical portion of laser placement is very step heavy, thus it was helpful to refer to a checklist to keep the procedure moving forward. During our dry run phases prior to implementation, specific step notations were included to help underscore procedural nuances ("Awl mark, remove grid, Beekley marker confirmation," "Align by hand to get close," "Insert ceramic stylet," "Measure and insert catheter with titanium stiffening stylet," etc.). Such personalized modifications to this checklist may be helpful to other groups as they individualize the checklist for their specific practice needs. Last, the specific time-point callouts in the procedural section of the checklist allowed us, in the initial cases, to focus on specific parts of the procedure that were causing delays. Moreover, the checklist was particularly helpful in alerting and reminding us to confirm our frame mount technique and rigid purchase.

Beyond the benefits in operative efficiency and patient safety, real-time MRI guidance can also facilitate laser repositioning if necessary. Unless real-time fluoroscopic imaging with $\mathrm{O}$-arm or computed tomography is completed prior to leaving the OR (as it was for all of our OR cases), repositioning the laser after MRI confirmation with the standard technique would necessitate a return to the OR for stereotactic repositioning. This would be a logistical challenge at most institutions, leaving the treating team with a decision to perform a potentially suboptimal ablation or abort the surgery. Repositioning was only deemed necessary in 1 of our 8 patients treated using the real-time MRI system, but this allowed for a more thorough ablation of mesial structures. While the benefits for robust ablations are a source of continued debate, ${ }^{5}$ the theoretical benefit of immediate stereotactic repositioning while in the MRI suite is one major advantage of a real-time MRI platform. It must also be noted that intraoperative or hybrid operative MRI systems can also provide similar benefits to the realtime MRI platform and should be used whenever possible for LITT. Although most institutions do not have these capabilities, they can adopt the real-time MRI platform for laser ablations in their diagnostic MRI suites. Last, at large centers where OR block time is often a limited resource, MRI operative suite capability provides an alternative option for stereotactic procedures. These substantial resources and procedural considerations with respect to the 2 different methodologies have been outlined in detail in Table 3. 
TABLE 3. Differences in methodology between OR and interventional MRI techniques

\begin{tabular}{|c|c|c|}
\hline Variable & OR & MRI \\
\hline OR & $\begin{array}{l}\text { Utilized for laser placement \& potentially on hold until imaging confir- } \\
\text { mation }\end{array}$ & $\begin{array}{l}\text { No need for OR except for rare emergency } \\
\text { complication }\end{array}$ \\
\hline Anesthesia & $\begin{array}{l}\text { Standard induction but switch on \& off transport monitors \& ventilation } \\
\text { for transport down to \& onto MRI-compatible anesthesia machines }\end{array}$ & $\begin{array}{l}\text { Nonstandard MRI suite location for induction, but } \\
\text { once intubated no further changes necessary }\end{array}$ \\
\hline Patient positioning & $\begin{array}{l}\text { Prone \& in Mayfield pins for laser placement, then out of pins \& supine } \\
\text { for transport \& in MRI }\end{array}$ & $\begin{array}{l}\text { Prone in ClearPoint 4-point fixation headframe } \\
\text { until procedure complete }\end{array}$ \\
\hline Laser placement & $\begin{array}{l}\text { With standard neuronavigation system (at our institution) or a stereo- } \\
\text { tactic frame }\end{array}$ & With real-time MRI platform \\
\hline Patient transport & Intubated from OR to MRI & None \\
\hline $\begin{array}{l}\text { Laser placement } \\
\quad \text { revision }\end{array}$ & $\begin{array}{l}\text { Transport back to OR from MRI (may not be feasible) or necessitates } \\
\text { intraoperative scan }\end{array}$ & Can revise immediately in MRI suite \\
\hline $\begin{array}{l}\text { Ablation revision w/ } \\
\text { alternate trajectory }\end{array}$ & Not possible w/o transporting back to OR & Can revise immediately in MRI suite \\
\hline
\end{tabular}

We experienced several hardware related technical difficulties during the adoption of real-time MRI for laser ablation that should be noted. First, the 1-piece sterile drape for the ClearPoint tower and MRI scanner can exert a significant amount of pull on the tower during movement of the MRI table if insufficient strain relief is provided. This is particularly true for prone cases, where the mounted tower is perpendicular to the floor. In one of our cases this led to dislodgement of the tower that necessitated replacing the tower completely. Second, adequate bony fixation for the ClearPoint tower is critical, as any movement can compromise trajectory accuracy. Utilization of longer screws may help overcome this challenge, but inadequate bony purchase must be recognized. Indeed, we encountered the highest target error in Case 7, and we found the tower to be loose at the time of inspection. Confirmation of adequate bony purchase is now a part of this procedure's checklist. At our institution, the MRI suite is commonly used for other sterile surgical procedures, thus, Stanford Infection Control protocols were already in place for terminal cleaning and sterilization of the MRI suite before and after the procedure as for any OR. Implementation of such protocols is of paramount importance at other institutions considering adopting the real-time MRI platform. Last, intracranial hemorrhage after laser placement is rare. ${ }^{14}$ Under standard protocol there should be a sterile OR that is prepared and available for emergencies to help facilitate immediate evacuation of a compressive hematoma. At our institution, an OR is always reserved for surgical emergencies, which would help mitigate any potential delay. Nevertheless, this is a risk of MRgLITT that must be considered, but it is not a risk specific to realtime MRI stereotaxy for LITT. In fact, the transfer delay from OR to MRI may have a greater impact on delaying the diagnosis of hematoma compared with a completely MRI-based procedure.

\section{Limitations}

This novel application of the real-time MRI platform for laser ablation is a preliminary study in a limited number of patients with brief follow-up. Thus, any results and conclu- sions warrant further prospective study with a much larger patient cohort, which is ongoing at our institution. This was a nonrandomized study with potential confounding variables. For example, trends in case time with the checklist could be confounded by a learning curve. While use of the checklist might have helped avoid an even greater learning curve without it, we cannot say with certainty this is true. However, the goal of the checklist was to anticipate this learning curve and maximize the rapid adoption of a complex, step-heavy new technique, and we believe our results reflect achievement of this goal. The checklist approach was not adopted for the traditional OR cases because the stereotactic techniques used for implanting the laser in the OR are standard at our institution, and common to depth electrodes, deep brain stimulation implants, and stereotactic and navigated biopsies. ${ }^{9}$ Our method for placement of the laser in the OR environment, involving Mayfield immobilization coupled with neuronavigation, was nearly identical to that used in the previously mentioned standard stereotactic surgeries, while laser placement in the MRI suite was completed with prone positioning without a Mayfield headframe and used the real-time MRI platform and MRI-compatible instruments for stereotactic laser placement. Thus, we do not believe that the operations are sufficiently similar to confer any type of "learning curve" benefit. However, given the sequential nature of our study, cases within the MRI cohort had the benefit of experience with laser ablation accumulated over all previous OR cases, and this may be at least partly responsible for the increased efficiency seen in our MRI cohort-though actual laser ablation durations were no different between cohorts, and there was no trend of increasing efficiency seen within the OR cohort (Fig. 4). On the contrary, there was an expected learning curve observed within our MRI cohort. In the OR cohort, there were many maneuvers involved in getting the patient from the OR to the MRI suite, including removal from Mayfield pins, repositioning and bed transfer, changing of anesthesia machines, transport, transfer into the MRI bore with supine positioning, taking care not to dislodge the laser fiber, and finally performing a confirmatory MRI scan. This translated into a significant 
but expected and necessary time delay before ablation was initiated (average 89 minutes). Given the risks of general anesthesia and the decreases seen in total and anesthesia times, our findings represent potential safety benefits of real-time MRI for laser placement and ablation. ${ }^{1}$

\section{Conclusions}

Adoption of a real-time MRI-guided stereotactic platform that uses MRgLITT for amygdalohippocampal ablation in the treatment of epilepsy can be achieved expediently. Completion of the entire procedure in the MRI suite may lead to improved procedure times, including total anesthesia time. Wider study and adoption of this method can lead to further refinements in technique and may provide a less invasive and safer option for patients undergoing LITT surgery.

\section{Acknowledgments}

We would like to gratefully acknowledge Dr. John Willie for providing input on our operative protocol. Matthew Rabon from MRI Interventions provided substantial assistance in the creation and adoption of our MRgLITT checklist. We are very appreciative for all of the MRI, anesthesia, and surgical personnel who provided invaluable support to the adoption of this novel technique at Stanford Health Care.

\section{References}

1. Beckmann U, Gillies DM, Berenholtz SM, Wu AW, Pronovost P: Incidents relating to the intra-hospital transfer of critically ill patients. An analysis of the reports submitted to the Australian Incident Monitoring Study in Intensive Care. Intensive Care Med 30:1579-1585, 2004

2. Connolly PJ, Kilpatrick M, Jaggi JL, Church E, Baltuch GH: Feasibility of an operational standardized checklist for movement disorder surgery. A pilot study. Stereotact Funct Neurosurg 87:94-100, 2009

3. Haynes AB, Weiser TG, Berry WR, Lipsitz SR, Breizat AHS, Dellinger EP, et al: A surgical safety checklist to reduce morbidity and mortality in a global population. N Engl J Med 360:491-499, 2009

4. Ho AL, Miller KJ, Cartmell S, Inoyama K, Fisher RS, Halpern CH: Stereotactic laser ablation of the splenium for intractable epilepsy. Epilepsy Behav Case Rep 5:23-26, 2016

5. Kang JY, Wu C, Tracy J, Lorenzo M, Evans J, Nei M, et al: Laser interstitial thermal therapy for medically intractable mesial temporal lobe epilepsy. Epilepsia 57:325-334, 2016

6. Kramer DR, Halpern CH, Buonacore DL, McGill KR, Hurtig HI, Jaggi JL, et al: Best surgical practices: a stepwise approach to the University of Pennsylvania deep brain stimulation protocol. Neurosurg Focus 29(2):E3, 2010

7. Kramer DR, Halpern CH, Connolly PJ, Jaggi JL, Baltuch $\mathrm{GH}$ : Error reduction with routine checklist use during deep brain stimulation surgery. Stereotact Funct Neurosurg 90:255-259, 2012

8. Larson PS, Starr PA, Bates G, Tansey L, Richardson RM, Martin AJ: An optimized system for interventional magnetic resonance imaging-guided stereotactic surgery: preliminary evaluation of targeting accuracy. Neurosurgery 70 (1 Suppl Operative):95-103, 2012
9. Miller K, Halpern $\mathrm{CH}$ : Stereotactic bony trajectory preservation for responsive neurostimulator lead placement following depth EEG recording. Cureus 8: 549, 2016

10. Miller KJ, Burns TC, Grant GA, Halpern CH: Responsive stimulation of motor cortex for medically and surgically refractive epilepsy. Seizure 33:38-40, 2015

11. Ostrem JL, Ziman N, Galifianakis NB, Starr PA, Luciano MS, Katz M, et al: Clinical outcomes using ClearPoint interventional MRI for deep brain stimulation lead placement in Parkinson's disease. J Neurosurg 124:908-916, 2016

12. Patel NV, Jethwa PR, Barrese JC, Hargreaves EL, Danish SF: Volumetric trends associated with MRI-guided laser-induced thermal therapy (LITT) for intracranial tumors. Lasers Surg Med 45:362-369, 2013

13. Patel P, Patel NV, Danish SF: Intracranial MR-guided laserinduced thermal therapy: single-center experience with the Visualase thermal therapy system. J Neurosurg 125:853860,2016

14. Pruitt R, Gamble A, Black K, Schulder M, Mehta AD: Complication avoidance in laser interstitial thermal therapy: lessons learned. J Neurosurg 126:1238-1245, 2017

15. Starr PA, Markun LC, Larson PS, Volz MM, Martin AJ, Ostrem JL: Interventional MRI-guided deep brain stimulation in pediatric dystonia: first experience with the ClearPoint system. J Neurosurg Pediatr 14:400-408, 2014

16. Torcuator RG, Hulou MM, Chavakula V, Jolesz FA, Golby AJ: Intraoperative real-time MRI-guided stereotactic biopsy followed by laser thermal ablation for progressive brain metastases after radiosurgery. J Clin Neurosci 24:68-73, 2016

17. Willie JT, Laxpati NG, Drane DL, Gowda A, Appin C, Hao $\mathrm{C}$, et al: Real-time magnetic resonance-guided stereotactic laser amygdalohippocampotomy for mesial temporal lobe epilepsy. Neurosurgery 74:569-585, 2014

18. Willie JT, Tung JK, Gross RE: MRI-guided stereotactic laser ablation, in Golby AJ (ed): Image-Guided Neurosurgery. Boston: Academic Press, pp 375-403, 2015

19. Wong JM, Ziewacz JE, Ho AL, Panchmatia JR, Bader AM, Garton HJ, et al: Patterns in neurosurgical adverse events: cerebrospinal fluid shunt surgery. Neurosurg Focus 33(5):E13, 2012

\section{Disclosures}

Dr. Keebaugh is a senior clinical application specialist at MRI Innovations.

\section{Author Contributions}

Conception and design: Halpern, Ho, Keebaugh, Drover, Grant. Acquisition of data: Ho, Sussman, Pendharkar, Le, Mantovani, Keebaugh. Analysis and interpretation of data: Halpern, Ho, Sussman, Pendharkar, Le, Mantovani, Keebaugh. Drafting the article: Ho, Sussman, Pendharkar, Keebaugh, Drover. Critically revising the article: Halpern, Ho, Sussman, Pendharkar, Keebaugh, Drover, Grant. Reviewed submitted version of manuscript: Halpern, Ho, Sussman, Le, Keebaugh, Drover, Grant, Wintermark. Statistical analysis: Ho. Administrative/technical/material support: Halpern, Ho, Grant, Wintermark. Study supervision: Halpern, Ho, Grant, Wintermark.

\section{Correspondence}

Casey H. Halpern, Department of Neurosurgery, Stanford University, 300 Pasteur Dr., A301, MC 5325, Stanford, CA 94305. email: chalpern@stanford.edu. 